\title{
Análise da nova metodologia da ISO 10545 para determinação da carga de ruptura e resistência a flexão de revestimentos cerâmicos
}

\author{
Aline Demarch ${ }^{1,2}$, Angela Waterkemper ${ }^{1,2}$, Djeisa Pasini², Fernando Zanelatto ${ }^{2}$, Sergio Ruzza ${ }^{2}$ \\ ${ }^{I}$ Universidade do Extremo Sul Catarinense - UNESC \\ Av. Universitária 1105, Bairro Universitário, Criciúma SC, Brasil, CEP 88806-000 \\ ${ }^{2}$ Eliane Revestimentos Cerâmicos, Cocal do Sul, SC, Brazil \\ *Rua Maximiliano Gaidzinski, 245 Centro, Cocal do Sul SC, Brasil, CEP 88845-000 \\ sergio.ruzza@eliane.com
}

\begin{abstract}
Resumo
A revisão da ISO 10545-4 sofreu alterações no procedimento de ensaio, para determinação da carga de ruptura e resistência a flexão de revestimentos cerâmicos. Na versão anterior o teste era realizado com amostras inteiras e na atual revisão, as amostras podem ou devem ser cortadas, dependendo do seu tamanho. Não se sabe porém se os resultados históricos se manterão após essa alteração. O trabalho consistiu em avaliar se as alterações impactam de forma significativa nos resultados de carga de ruptura e resistência a flexão dos produtos. Foi realizada uma análise comparativa do desempenho do produto no teste de carga de ruptura e resistência a flexão, com amostras inteiras e cortadas, de acordo com a nova especificação da ISO 10545-4. A análise comparativa entre resultados, foi realizada por meio do teste "t" de Student, com peças de diferentes formatos e de diferentes tipologias: monoporosa, monoqueima e porcelanato esmaltado. As análises mostraram que, há diferenças entre os resultados obtidos com procedimento antigo e com o procedimento novo, tanto para a carga de ruptura quanto para a resistência a flexão. Portanto, fica confirmado, com 95\% de confiança, que existem diferenças entre os resultados obtidos com amostras inteiras e amostras cortadas.
\end{abstract}

Palavras Chave: Carga de Ruptura, Resistência a Flexão

\section{Introdução}

O teste de flexão é o método mais comum usado para medir a resistência à tração uniaxial de cerâmicas [1]. A revisão da ISO 10545:2019-4 [2] trouxe diversas alterações no procedimento para determinação na carga de ruptura e resistência a flexão, em especial no que diz respeito ao corte das amostras a serem ensaiadas. Atualmente a NBR 13818 [3], assim como na antiga ISO 10545:2014-4 [4], especifica que se deve, sempre que possível, ensaiar peças inteiras, porém, a nova revisão da ISO 10545-4 [2] estabelece para alguns casos, que as amostras devem ser obrigatoriamente cortadas.

Até o momento, não se tem conhecimento de como esta alteração irá influenciar nos resultados de carga de ruptura e resistência a flexão. Com isso, é necessário avaliar se os produtos irão atender as especificações de carga de ruptura e resistência a flexão após a implantação deste novo método.

O trabalho em questão, tem o objetivo de verificar estatisticamente, se as alterações no procedimento irão influenciar nos resultados do ensaio, ou seja, avaliar se as modificações impactam de forma significativa nos resultados de carga de ruptura e resistência a flexão dos produtos. As propriedades de resistência das cerâmicas são geralmente dispersas, portanto, uma análise estatística é indispensável para o entendimento da caracterização mecânica deste material. [5]
Fratura de materiais frágeis (por exemplo, cerâmica) geralmente inicia a partir de falhas [6], que são distribuídas no material. A força que a amostra resiste depende do tamanho da falha principal, que varia de amostra para amostra. Portanto, a resistência de materiais frágeis tem que ser descrita por uma função de probabilidade (estatística) [7], [8], [9].

Os materiais cerâmicos apresentam uma série de defeitos que podem atuar como elementos concentradores de tensões e que determinam os pontos onde se inicia a fratura do produto. A resistência mecânica de um produto depende de sua microestrutura e, principalmente, da distribuição e tamanho dos defeitos presentes. Como esta distribuição é quase sempre aleatória, a resistência avaliada experimentalmente apresenta uma dispersão. Para obter a resistência mecânica experimentalmente não é suficiente apresentar somente o valor médio, mas é essencial levar em consideração a dispersão dos resultados [10].

O corte das peças pode atuar como um concentrador de defeitos, ou como uma forma de alívio de tensões presentes na peça, esses dois fatores devem interferir diretamente no desempenho do produto, quando este for submetido a uma força axial, alterando, portanto, os valores de carga de ruptura e resistência a flexão das peças analisadas. 


\section{Procedimento Experimental}

Os testes foram realizados com 30 peças de cada tamanho, para condição sem corte (peças inteiras) e mais 30 peças de cada tamanho, para a condição, com corte. A tabela 01 mostra os formatos testados, a espessura padrão, a classificação de grupo de absorção e em quais dimensões foram extraídas as amostras, para o teste com amostras cortadas:

Foram selecionados estes tamanhos, tendo em vista que a norma 10545:2019-4 [2] divide os produtos em dois grupos: produtos com espessura menor que $7,5 \mathrm{~mm}$ e maior ou igual a $7,5 \mathrm{~mm}$. O intuito foi selecionar amostras com espessuras distintas, que se encaixasse nestes dois grupos.

Para as peças que possuem espessura maior ou igual a $7,5 \mathrm{~mm}$, a norma permite que seja realizado o corte das mesmas, desde que um dos lados seja maior que $600 \mathrm{~mm}$, entretanto, a referida norma também estabelece que sempre deve-se preferir resultados com amostras inteiras. Se a peça possuir espessura menor que $7,5 \mathrm{~mm}$, as peças devem obrigatoriamente ser ensaiadas com amostras cortadas com dimensões de 200 x $200 \mathrm{~mm}$, quando a área da peça estiver entre $40.000 \mathrm{~mm}^{2}$ e $360.000 \mathrm{~mm}^{2}$.

O equipamento utilizado para os testes foi o crômetro digital, marca Servitech com capacidade para ensaiar peças de até $1200 \mathrm{~mm}$ de largura e comprimento. A espessura da seção de ruptura foi determinada com paquímeto digital marca Mitutoyo.

Após os testes foi realizada a análise estatística dos resultados, utilizando o teste estatístico " $\mathrm{t}$ " para as duas amostras, com objetivo de avaliar com $95 \%$ de significância, se existe diferença entre os resultados dos ensaios com amostras inteiras e com amostras cortadas para cada formato testado.

\section{Resultados e Discussões}

A seguir, serão apresentados os resultados com valores médios e individuais de carga de ruptura e a análise estatística por meio do teste " $t$ " de Student.

\subsection{Resultados Carga de Ruptura e Resistência a flexão}

As figuras a seguir mostram os resultados individuais de cada uma das 30 amostras testadas na condição cortada e inteira, sendo esses resultados individuais indicados pelos pontos coloridos. Os pontos cinza indicam o valor médio das 30 amostras testadas. Serão apresentados apenas os resultados de carga de ruptura pois os resultados de resistência a flexão apresentaram comportamento semelhante, em termos de variabilidade e média quando se compara amostras cortadas com as amostras inteiras.
A figura 01 mostra os resultados do formato 450x450x6,5 mm pertencente ao grupo de absorção BIIb. Neste gráfico, é possível perceber que a variabilidade dos resultados com as amostras cortadas é maior do que com as amostras inteiras, indicada pela amplitude dos pontos coloridos. Este fato pode ser explicado devido a possível fragilização e alívio das tensões internas ocorrido no momento do corte das peças.

O resultado médio das amostras cortadas foi inferior ao resultado médio das amostras inteiras, indicado pelos pontos em cinza. Algumas amostras individuais ficaram próximas ao limite estipulado pela norma de especificação ISO 13006:2018 [12] para produtos BIIb, com espessura menor que $7,5 \mathrm{~mm}$, de $500 \mathrm{~N}$.

O comportamento da resistência a flexão foi semelhante ao de carga de ruptura no que diz respeito a resultados médios e variabilidade.

A figura 02 mostra os resultados do formato $300 \times 400 \times 6,5 \mathrm{~mm}$, pertencente ao grupo BIII. Também se

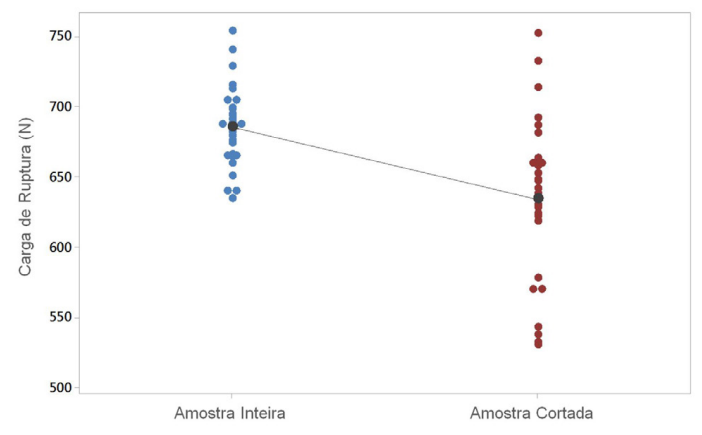

Figura 01 - Resultados individuais de Carga de Ruptura da bitola 450x450x6,5 mm, pertencente ao grupo de absorção BIIb.

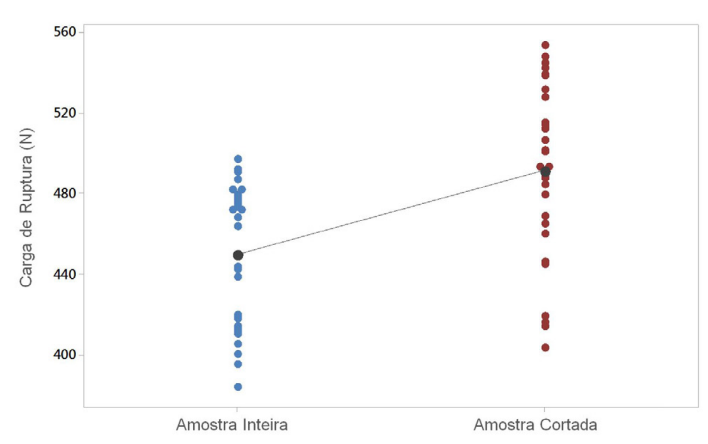

Figura 02 - Resultados individuais de Carga de Ruptura da bitola 300x400x6,5 mm, pertencente ao Grupo da Absorção BIII.

Tabela 01 - Amostras utilizadas no teste

\begin{tabular}{ccccc}
\hline Tamanho $(\mathbf{m m})$ & Área $\left(\mathbf{m m}^{\mathbf{2}}\right)$ & Espessura $(\mathbf{m m})$ & Formato cortado $(\mathbf{c m})$ & Classificação \\
\hline 902x902 & 813.604 & 11 & $60 \times 60$ & Porcelanato Esmaltado \\
$590 \times 1182$ & 697.380 & 11 & $30 \times 60$ & Porcelanato Esmaltado \\
$194 \times 1182$ & 229.308 & 11 & $20 \times 60$ & Porcelanato Esmaltado \\
$450 \times 450$ & 202.500 & 6,5 & $20 \times 20$ & BIIb \\
$450 \times 902$ & 405.900 & 10,5 & $30 \times 60$ & BIII \\
$300 \times 400$ & 120.000 & 6,5 & $20 \times 20$ & BIII \\
\hline
\end{tabular}


observou uma dispersão maior dos resultados individuais quando as peças foram cortadas, sendo que, neste caso, o valor médio das amostras cortadas foi superior ao valor médio das amostras inteiras.

Todas as amostras ficaram acima do limite estipulado de carga de ruptura, pela norma de especificação ISO 13006:2018 [12] para produtos BIII, de espessura menor que $7,5 \mathrm{~mm}$, de $200 \mathrm{~N}$.

O comportamento da resistência a flexão foi semelhante ao de carga de ruptura no que diz respeito a resultados médios e variabilidade.

A figura 03 mostra os resultados do formato 590x1182x11 mm, pertencente ao grupo de absorção BIa, com uma dispersão semelhante para ambas condições e um resultado médio com as amostras cortadas superior aos resultados com amostras inteiras.

Todas as amostras, nas duas condições, ficaram acima do limite de $1500 \mathrm{~N}$ estipulado de carga de ruptura, pela norma brasileira de especificação de porcelanatos, NBR 15463 [11], para espessura maior ou igual a 7,5 mm.

Embora a amostra deste tamanho indicou comportamento da resistência a flexão semelhante ao de carga de ruptura, para algumas amostras individuais, do teste com a amostra inteira, o resultado de resistência a flexão foi inferior ao especificado pela NBR 15463 [11] de $37 \mathrm{MPa}$. Enquanto que para condição cortada, nenhuma amostra apresentou resultado de resistência a flexão inferior ao especificado.

A figura 4 mostra os resultados individuais e médio, do formato $194 \times 1182 \times 11 \mathrm{~mm}$, pertencente ao grupo de absorção BIa. É possível perceber a grande variabilidade dos resultados tanto para as amostras cortadas como para as amostras inteiras, porém o resultado médio das amostras cortadas foi maior.

Todas as amostras ficaram acima do limite estipulado de carga de ruptura, pela norma brasileira de especificação de porcelanatos, NBR 15463 [11], para espessura maior ou igual a $7,5 \mathrm{~mm}$, de $1500 \mathrm{~N}$.

Os resultados de resistência a flexão apresentaram comportamento semelhante ao da carga de ruptura.

A figura 05 mostra os resultados individuais e médio, do formato $902 \times 902 \times 11 \mathrm{~mm}$, sendo o valor médio nas duas situações, com amostras cortadas e amostras inteiras, muito próximos. Porém os valores individuais, mostra que a variabilidade dos resultados com as amostras cortadas é muito maior quando comparado com amostras inteiras.

Todas as amostras ficaram acima do limite estipulado de carga de ruptura, pela norma brasileira de especificação de porcelanatos, NBR 15463 [11], para espessura maior ou igual a $7,5 \mathrm{~mm}$, de $1500 \mathrm{~N}$.

O mesmo também ocorre com a resistência a flexão, os valores médios são muito próximos enquanto que os valores individuais se mostram muito mais dispersos na amostra testada obtida por corte.

A figura 06 mostra os resultados individuais e médio, do formato 450x902x10,5 mm, sendo possível visualizar que o resultado médio foi superior com as amostras cortadas. Em relação a variabilidade dos resultados, apresentou semelhança quando comparado os resultados das amostras cortadas e amostras inteiras.

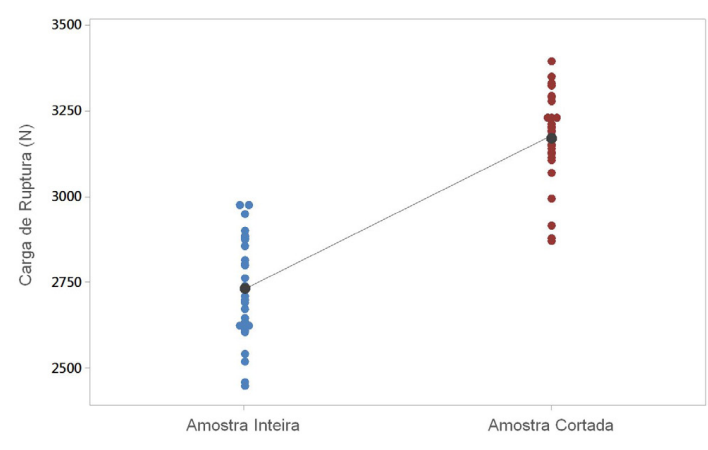

Figura 03 - Resultados individuais de Carga de Ruptura da bitola 590x1182x11 mm, pertencente ao grupo de absorção BIa.

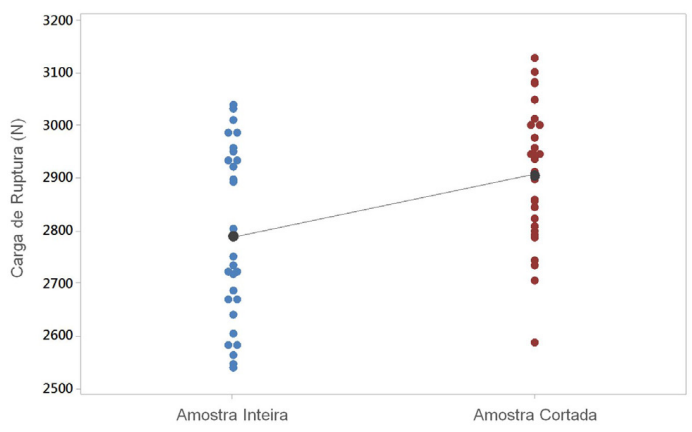

Figura 04 - Resultados individuais de Carga de Ruptura da bitola 194x1182x11 mm, pertencente ao grupo de absorção BIa.

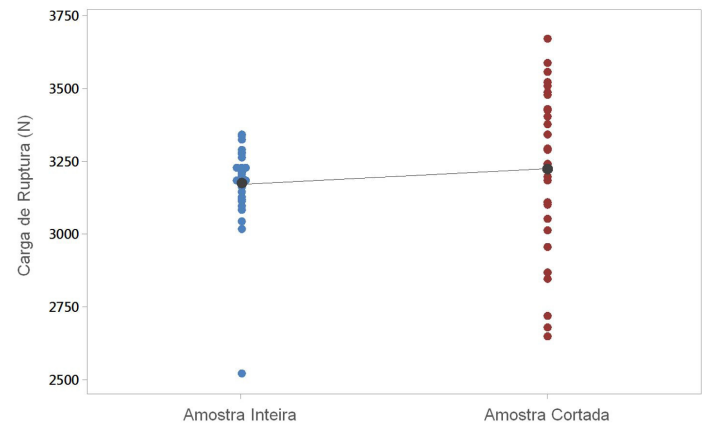

Figura 05 - Resultados individuais de Carga de Ruptura da bitola $902 \times 902 \times 11 \mathrm{~mm}$, pertencente ao grupo de absorção BIa.

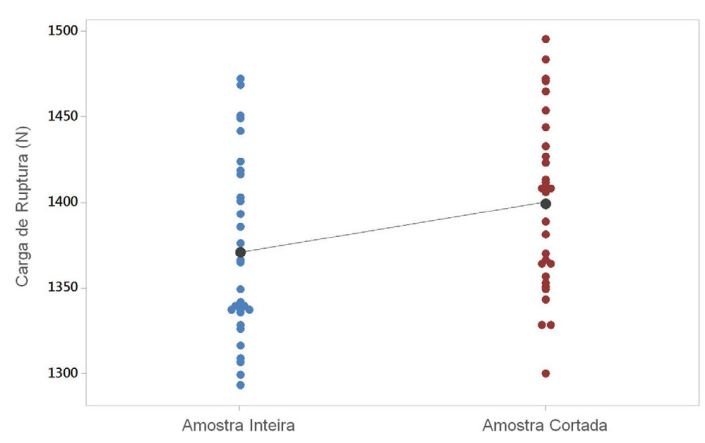

Figura 06 - Resultados individuais de carga de ruptura da bitola 450x902×10,5mm, pertencente ao grupo de absorção BIII. 
Todas as amostras apresentaram resultados superiores a especificação da norma ISO 13006:2018 [12] para produtos BIII, de espessura maior ou igual que $7,5 \mathrm{~mm}$, de $600 \mathrm{~N}$.

Os resultados de resistência a flexão se mostraram muito semelhantes e muito próximos tanto nos valores individuais quanto no valor médio, quando comparado resultados com amostras cortadas e amostras inteiras.

\subsection{Análise Estatística}

A análise estatística foi realizada pelo teste " $t$ " de Student, realizado para um nível de significância de 95\%, que avalia a diferença entre os resultados das amostras inteiras e amostras cortadas. Quando o valor de " $p$ " for inferior a 0,05 pode-se afirmar com $95 \%$ de confiança que os resultados são diferentes, ou seja, não podem ser considerados estatísticamente iguais.

A tabela 02 mostra os resultados do teste " $t$ " realizado com os resultados dos testes de carga de ruptura, confirmando para todos os formatos, que o ensaio de de carga de ruptura das amostras cortadas e amostras inteiras são diferentes, ou seja, não podem ser considerados estatisticamente iguais. Todos os valores de " $p$ " apresentaram valores inferiores a 0,05 , então pode-se confirmar com $95 \%$ de confiança que existem diferenças entre o resultado obtido com amostras inteiras e amostras cortadas.

Deve-se salientar que a revisão de ISO 10545:2019-4 especifica a obrigatoriedade de corte dos formatos $450 \times 450 \mathrm{~mm}$ e $300 \times 400 \mathrm{~mm}$, pois possuem espessura menor que 7,5 mm área entre $40.000 \mathrm{~mm}^{2}$ e $360.000 \mathrm{~mm}^{2}$.

Quanto aos demais tamanhos, a norma permite que as amostras sejam cortadas caso não se tenha equipamento com capacidade para ensaiar peças inteiras, mas estabelece que os resultados com peças inteiras sempre devem ser preferidos. Portanto, o ideal é realizar os testes com peças inteiras.

A tabela 03, mostra os resultados do teste "t" para o ensaio de resistência a flexão, neste caso os valores de "p" para todos os tamanhos foram inferiores a 0,05 , com exceção do formato 450x902 mm, em que o valor de "p" foi de 0,180 . Portanto, pode-se confirmar com $95 \%$ de certeza que há diferenças entre o resultados obtidos com amostras inteiras e amostras cortadas para os formatos testados, exceto o formato 450x902 mm.

A figura 07 mostra o comparativo dos resultados de carga de ruptura de cada um dos tamanhos testados. Visualmente é possível distinguir uma diferença entre os resultados de amostras inteiras e amostras cortadas, embora os resultados médios ficaram próximos em alguma amostras, pela análise estatística foi comprovado que os resultados não podem ser considerados estatísticamente iguais.

Se os resultados das amostras estiverem próximo ao limite de especificação da norma, essa diferença de metodologia poderá provocar a aprovação ou reprovação do produto, devido a variabilidade dos resultados demonstrados anteriormente.

Os resultados de resistência a flexão da figura 08 , seguem o mesmo comportamento dos resultados de carga de ruptura. Apenas a bitola 450x902 se destaca pelo fato
Tabela 02 - Teste "t" para a carga de ruptura

\begin{tabular}{ccl}
\hline Formato $(\mathbf{m m})$ & Valor-T & Valor-p \\
\hline $450 \times 450$ & 4,40 & $7,29.10^{-05}$ \\
$300 \times 400$ & $-4,13$ & $1,24.10^{-04}$ \\
$590 \times 1182$ & $-12,18$ & 0,000 \\
$194 \times 1182$ & $-3,09$ & 0,0031 \\
$902 \times 902$ & $-0,81$ & $4,20.10^{-01}$ \\
$450 \times 902$ & $-2,08$ & 0,042 \\
\hline
\end{tabular}

Tabela 03 - Teste "t" para a resistência a flexão

\begin{tabular}{ccl}
\hline Formato $(\mathbf{m m})$ & Valor-T & Valor-p \\
\hline $450 \times 450$ & 3,31 & $1,78.10^{-03}$ \\
$300 \times 400$ & $-6,43$ & $6,48.10^{-08}$ \\
$590 \times 1182$ & $-11,10$ & $1,20.10^{-15}$ \\
$194 \times 1182$ & $-2,67$ & 0,01 \\
$902 \times 902$ & $-0,88$ & $3,82.10^{-01}$ \\
$450 \times 902$ & $-1,36$ & 0,180 \\
\hline
\end{tabular}

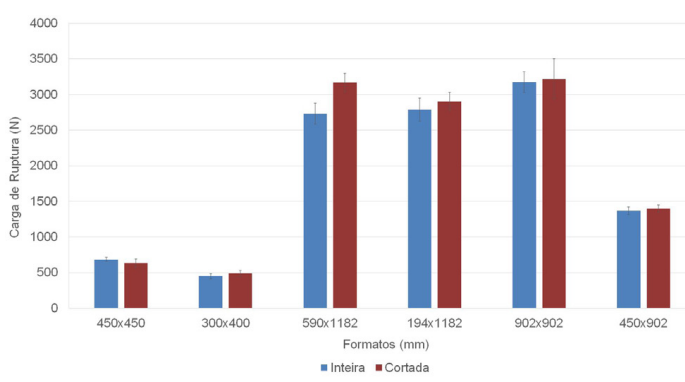

Figura 07 - Resultados médio de carga de ruptura de cada bitola testada

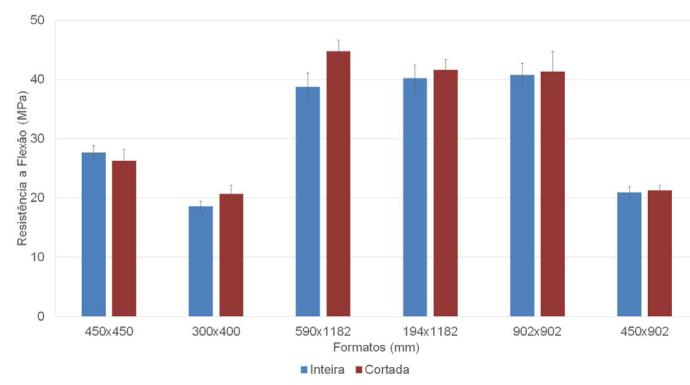

Figura 08 - Resultados médio de resistência a flexão de cada bitola testada

de ter apresentado valor médio de resistência a flexão, muito próximo entre amostras cortadas e inteiras, e também desvio padrão muito semelhante, fato comprovado pelo teste " $t$ " em que se obteve um valor " $p$ " superior a 0,05 , conforme já discutido anteriormente.

\section{Conclusões}

Os resultados apresentados indicam que não é possível substituir o procedimento indicado pela ISO 10545-4, para o grupo de produtos com espessura menor que $7,5 \mathrm{~mm}$, 
onde é obrigatório o corte das peças para a realização dos ensaios. Com isso, sempre que a norma indicar que é obrigatório realizar o corte das peças, esse corte de fato, deverá ser realizado, pois como também comprovado com o teste " $t$ ", não se pode considerar estatísticamente iguais os resultados das peças cortadas e das peças inteiras.

Quanto ao grupo de produtos com espessura maior que 7,5 mm a ISO 10545-4 estabelece que sempre deve-se preferir resultados de testes realizados com peças inteiras, tendo em vista essa descrição, os testes devem continuar sendo realizados com peças inteiras, uma vez que as análises estatísticas, por meio do teste " $t$ ", comprovaram que os resultados são estatisticamente diferentes. Outro ponto a se destacar nesse grupo, é que os valores médios foram sempre maiores nos testes realizados com as peças cortadas, tanto para a carga de ruptura quanto para a resistência a flexão.

\section{Referência}

[1] QUINN, George D. et al. Flexural Strength of Ceramic and Glass Rods. Journal Of Testing And Evaluation, West Conshohocken, v. 37, p.1-23, abr. 2009.

[2] International Organization for Standardization. ISO 10545 Part 4 - Determination of modulus of rupture and breaking strength. 2019, Suíça. 8 p.

[3] Associação Brasileira de Normas Técnicas. NBR 13818 Anexo C - Determinação da Carga de Ruptura e Módulo de Resistência a Flexão - Especificações e Métodos de Ensaios. Rio de Janeiro, 1997. 14 p.
[4] International Organization for Standardization. ISO 10545 Part 4 - Determination of modulus of rupture and breaking strength. 2014, Suíça. 5 p.

[5] Ćurković, Lidija \& Bakić, A \& Kodvanj, Janos \& Haramina, Tatjana. (2010). Flexural strength of alumina ceramics: Weibull analysis. Transactions of FAMENA. 34. 13-19.

[6] Evans AG. Structural reliability: a processing-dependent phenomenon. J Am Ceram Soc 1982;65:127-37.

[7] Davidge RW. Mechanical behaviour of ceramics. Cambridge: Cambridge University Press; 1979.

[8] Danzer R. Mechanical performance and lifetime prediction. In: Brook RJ, editor. Concise encyclopedia of advanced ceramic materials. Pergamon Press; 1991. p. 286-99.

[9] DANZER, R. et al. Fracture statistics of ceramics Weibull statistics and deviations from Weibull statistics. Engineering Fracture Mechanics, [s.1.], v. 74, n. 18, p.29192932, dez. 2007. Elsevier BV. http://dx.doi.org/10.1016/j. engfracmech.2006.05.028.

[10] MARGARIDO, A. P. ou MENEGAZZO, A.P.M.; PASCHOAL, José Octavio Armani ; ANDRADE, Ariane Mainetti de ; CARVALHO, Júlio Cesar; GOUVÊA, Douglas . Avaliação da resistência mecânica e módulo de weibull de produtos tipo grês porcelanato e granito. Cerâmica Industrial, São Carlos/SP, v. 7, p. 24-32, 2002.

[11] Associação Brasileira de Normas Técnicas. NBR 15463 Placas Cerâmicas para Revestimento - Porcelanato. Rio de Janeiro, 2013. 7 p.

[12] International Organization for Standardization. ISO 13006 Ceramic tiles - Definitions, classification, characteristics and marking. 2018, Suíça. 53 p. 